\title{
Inflammatory T-lymphocyte proliferation in morbid obesity
}

\author{
K Van der Weerd ${ }^{1 *}$, P M van Hagen 1,2, W A Dik¹, B S Schrijver ${ }^{1}$, D H Schweitzer ${ }^{3}$, A W Langerak ${ }^{1}$, R M Kiewiet ${ }^{2}$, \\ M O Van Aken ${ }^{4}$, JJM Van Dongen ${ }^{1}$, A J Van der Lelij ${ }^{2}$, FJT Staal ${ }^{1,5}$ \\ From 5th European Workshop on Immune-Mediated Inflammatory Diseases \\ Sitges-Barcelona, Spain. 1-3 December 2010
}

\section{Introduction}

Obesity is one of the leading causes of preventable death in the Western world, and its prevalence is dramatically increasing. Although obesity is primarily a metabolic disease, immunological aberrations also significantly contribute to obesity-related morbidity. The well-established state of chronic low-grade inflammation is considered to be crucial in the development of complications such as diabetes and atherosclerosis. Moreover, obesity is associated with increased susceptibility to infections, cancer and autoimmunity. Thus, disturbed immune regulation may exist in obese individuals. However, until now, a detailed description of systemic immunological changes in obesity is lacking.

\section{Aim}

We studied the effects of obesity on the T-cell compartment of morbid obese subjects.

\section{Patients and methods}

Blood was collected from eight morbid obese subjects and twenty lean control subjects. The T-cell compartment was defined phenotypically using flow cytometry and molecularly by T-cell receptor excision circle (TREC) analyses.

\section{Results}

We found a selective increase in T-lymphocyte number in morbid obese subjects, which was mainly caused by an increase in $\mathrm{CD}^{+} \mathrm{T}$-lymphocytes. Also, in morbid obese subjects TREC content was decreased in all T-lymphocyte subpopulations, demonstrating that the increase in T-lymphocytes is mainly caused by increased proliferation. Moreover, in morbid obese subjects we found increased plasma levels of IL-7 and CCL5, both potent enhancers of T-lymphocyte proliferation. Also, plasma of morbid obese subjects enhanced T-lymphocyte proliferation in vitro.

Finally, both $\mathrm{CD}^{+}$and $\mathrm{CD} 8^{+} \mathrm{T}$-lymphocytes had some skewing of the TCR repertoire.

\section{Conclusion}

These new data demonstrate that morbid obesity is associated with an increase in mainly CD4+ T-lymphocytes. Most likely this is related to increased plasma levels of cytokines that stimulate T-lymphocyte proliferation. This increase in T-lymphocytes may contribute to the increased risk of autoimmunity and cancer described in morbid obesity.

\section{Author details}

${ }^{1}$ Dept. of Immunology, Erasmus MC, Rotterdam, The Netherlands. ${ }^{2}$ Dept. of Internal Medicine, Erasmus MC, Rotterdam, The Netherlands. ${ }^{3}$ Dept. of Internal Medicine, Reinier de Graaf Hospital, Delft, The Netherlands. ${ }^{4}$ Dept. of Internal Medicine, HagaZiekenhuis, Den Haag, The Netherlands. ${ }^{5}$ Dept. of Immunohematology and Blood Transfusion, Leiden University Medical Center, Leiden, The Netherlands.

Published: 25 November 2010

\section{doi:10.1186/1479-5876-8-S1-P12}

Cite this article as: Van der Weerd et al:: Inflammatory T-lymphocyte proliferation in morbid obesity. Journal of Translational Medicine 2010 8(Suppl 1):P12.

${ }^{1}$ Dept. of Immunology, Erasmus MC, Rotterdam, The Netherlands

Full list of author information is available at the end of the article 